\title{
Organizational Contact Title
}

National Cancer Institute

\section{Source}

National Cancer Institute. Organizational Contact Title. NCI Thesaurus. Code C93882.

A descriptive or distinctive appellation, especially one belonging to a person by right of rank, office, attainment, etc. 